\title{
Evaluación en educación a distancia por confinamiento
}

\author{
Assessment in distance education by confinement \\ Efrén Viramontes Anaya \\ Otoniel Viramontes Campos
}

\begin{abstract}
RESUMEN
Este documento es una reflexión desde la perspectiva humanista sobre la evaluación de los aprendizajes en situación de contingencia, originada por la emergencia sanitaria, que obligó a los y las estudiantes del todo el sistema educativo mexicano al confinamiento en sus hogares y a desarrollar las actividades escolares a distancia. Se analizan los problemas en la enseñanza y el aprendizaje en el contexto de México, en los que la evaluación tiene una función muy importante. Se describe cómo, en medio de la incertidumbre, las instituciones y los docentes han realizado una serie de acciones para adecuar la evaluación a estas condiciones de improvisación y de toma de decisiones emergentes, que en su mayoría pretenden atender las necesidades de acreditar y certificar los aprendizajes, así como a resarcir las afectaciones derivadas por el encierro y las limitaciones para atender las exigencias del sistema educativo en la intención de salvar los ciclos escolares. A la par de la reflexión y el análisis se proponen acciones concretas que favorecen la realización de una evaluación más adecuada, justa, flexible e incluyente.
\end{abstract}

Palabras clave: aprendizaje, enseñanza, evaluación del estudiante, pandemia.

\begin{abstract}
This document is a reflection, from the humanist perspective, on the evaluation of learning in a contingency situation, originated by the health emergency, which forced students throughout the Mexican educational system to be confined in their homes and to carry out school activities at a distance. It analyzes the problems in teaching and learning, in which evaluation plays an especially important role. It describes how, in the midst of uncertainty, institutions and teachers have carried out a series of actions to adapt evaluation to these conditions of improvisation and emerging decision making, most of which are intended to meet the needs of accrediting and certifying learning, as well as to compensate for the effects derived from confinement and the limitations to meet the demands of the educational system with the intention of saving school cycles. Along with the reflection and analysis, concrete actions are proposed that encourages the realization of a more adequate, fair, flexible, and inclusive evaluation.
\end{abstract}

Keywords: learning, teaching, student assessment, pandemic. 


\section{INTRODUCCIÓN}

En tiempos de contingencia, por lo general las actividades de enseñanza y aprendizaje se tornan más complicadas que en condiciones de normalidad. En este contexto no escapan del análisis y de la preocupación de docentes y autoridades educativas, pues de ellas depende la valoración del avance y cumplimiento de los programas educativos, sobre todo de la acreditación y certificación de los logros de aprendizaje. Pero, más allá del asunto administrativo, es todavía más complejo el hecho de realizar una evaluación justa para la variedad de estudiantes y sus condiciones personales, emocionales, de salud y socioeconómicas.

La evaluación del aprendizaje siempre ha sido un tema de gran interés para quienes nos dedicamos a la docencia, porque es una parte muy importante del planteamiento del proceso de enseñanza y aprendizaje, por ello invariablemente está presente, de manera consciente o inconsciente por los docentes, alumnos, directivos escolares y padres de familia, en todo evento educativo formal.

El propósito de este ensayo es el de reflexionar sobre las situaciones de evaluación del aprendizaje en el contexto mexicano de la educación a distancia establecida por la emergencia sanitaria. Para adentrarnos a este tema es indispensable conceptualizar este término, que tiene implicaciones de diversa índole, desde las académicas hasta las psicológicas y sociales, en el que se involucran aspectos tan diversos que, si no nos percatamos de ellos, podríamos caer en situaciones de inequidad y de imprecisiones al momento de establecer una valoración de los procesos y avances de los aprendizajes de los estudiantes.

La evaluación basada en la medición es un tópico que ha quedado superado en la literatura académica sobre esta temática, en la que generalmente se realiza una comparación entre estos dos términos: evaluación y medición. Queda claro que la evaluación no es estrictamente una medición, y que no la excluye, sino que implica otros elementos cualitativos que ayudan a tener un acercamiento más profundo que permiten tener una valoración de los aprendizajes de manera más adecuada.

Efrén Viramontes Anaya. Profesor-investigador de tiempo completo en la Escuela Normal Rural Ricardo Flores Magón, Saucillo, Chihuahua, México. Es maestro en Educación campo Práctica Docente por la Universidad Pedagógica Nacional y doctor en Ciencias de la Educación por el Instituto Pedagógico de Estudios de Posgrado. Reconocido por el Sistema Nacional de Investigadores, perfil Prodep, miembro de la Red de Investigadores Educativos Chihuahua y presidente de la Red Nacional de Formación Docente (2015 al 2019). Las aportaciones académicas principales han sido en torno la formación docente, enseñanza y aprendizaje del lenguaje, y evaluación. Correo electrónico: efren8000@hotmail.com. ID: https://orcid.org/0000-0003-4378-3095.

Otoniel Viramontes Campos. Profesor-investigador de tiempo completo en la Escuela Normal Rural Ricardo Flores Magón, Saucillo, Chihuahua, México. Es maestro en Educación campo Práctica Docente por la Universidad Pedagógica Nacional. Sus aportaciones académicas principales han sido en torno la formación docente, inclusión y evaluación. Forma parte del cuerpo académico en consolidación Didácticas Especializadas y Políticas Educativas -ENRRFM-CA-5. Correo electrónico: leprechu@, gmail.com. ID: https://orcid.org/0000-0002-0153-5846. 
En los análisis que se han realizado sobre la evaluación, de acuerdo con Nápoles (2020), se hace muy evidente lo que concluye al respecto cuando señala que "lo hacemos mal (de forma y de fondo), [si se considera como] comprobación de resultados de aprendizaje" (p. 5). Esta forma de evaluar ha sido muy frecuentemente aplicada. Con esta idea se hace una separación del proceso de enseñanza y el de la evaluación, se enseña y posteriormente se evalúa. Se ha evitado sistemáticamente realizar un proceso simultáneo que ayude al docente a irse percatando conscientemente, sobre la marcha de la enseñanza, de los indicadores que le hacen saber qué tanto se va aprendiendo, quiénes sí lo hacen, quiénes están teniendo problemas para ello, cuáles son los elementos específicos del contenido educativo (considerado en forma genérica que incluye las intenciones educativas) que no se están logrando. Esto coincide completamente con la propuesta de Casanova (2020), quien sugiere una evaluación de procesos en un ambiente inclusivo.

Una conceptualización frecuente y acertada es en la que coinciden Miller (2020), De la Oliva (2020) y Casanova (2020) al conceptualizarlo como un proceso sistemático de acopio de información sobre el avance y forma en que se da el aprendizaje, seguido de una valoración con la intención de mejorar la consecución de las intenciones educativas que se pretende desarrollar en el alumnado. Este tipo de conceptos se consideran mejores si se dirigen hacia la recogida de información de los procesos de aprendizaje sin desatender las condiciones sociales y personales en que se realizan.

En lo que se refiere a los aspectos psicológicos y sociales, se rescatan las aportaciones de Ruiz (2020) y Requena (2020), que señalan la necesidad de considerar los aspectos socioemocionales para realizar procesos evaluativos más acordes a las necesidades y condiciones personales y contextuales de los estudiantes, basados en un enfoque complejo en el que se considera una serie de elementos que interactúan de manera dinámica y multidireccional en un esquema sistémico interconectado. Este enfoque exige de los docentes una perspectiva holística, incluyente, propositiva, crítica y empática con el alumnado.

Para cerrar con esta parte de la conceptualización de la evaluación, se habrá de considerar a la evaluación como un proceso dinámico insertado en un sistema complejo de personas, contexto y acciones, en el que la comparación y la valoración holística son elementos fundamentales del proceso, en el que la consideración de los referentes son el punto crítico de comparación entre los sujetos, sus condiciones y las intenciones educativas formales del sistema educativo.

En lo que corresponde a las funciones de la evaluación, estas han sido tratadas ampliamente por Casanova (1998) al señalar tres tipos: diagnóstica, formativa y sumativa, coincidentes con los momentos inicial, procesual y final. Todas deben usarse, especialmente la formativa. 
Por otra parte, Tobón (2020, citado en Ruiz, 2020), explica que la evaluación tiene varias funciones basadas en cuatro constructos:

...diagnostica, retroalimenta y logra la mejora continua en la formación integral mediante el seguimiento, el apoyo y la colaboración y debe considerar 6 ejes: 1) resultados de aprendizaje, 2) problema y producto, 3) instrumento, 4) valoración, 5) mejora y 6) socialización [...] la valoración de la evaluación se basa en cuatro constructos que deben orientar la estrategia del docente como el saber ser, saber hacer, saber conocer y saber convivir, que, por su valor formativo trasciende y va a configurar las necesidades del estudiante [p. 2].

Esta aportación de Tobón (2020) se fundamenta en la enseñanza y aprendizaje basado en competencias, cuya metodología cambia cuando se inscribe en enfoques más avanzados como los que se proponen por la UNESCO (2013) con respecto al desarrollo de capacidades, o las que se establecen por medio de la identificación y solución de ejes problemáticos situados en la realidad de los estudiantes. Este último tipo de enfoque es el que parece ser más adecuado para los procesos de contingencia en que el aprendizaje se efectúa en actividades a distancia, que implica la aplicación de un paradigma diferente, en la enseñanza y en la evaluación, con respecto a la realización de las clases presenciales, que representan el paradigma más común en el trabajo escolarizado.

La principal función de la evaluación se resume en el uso de esta para mejorar los aprendizajes de los estudiantes, a la vez que se revisa también la eficacia del proceso de enseñanza del docente. Los insumos que aporta la evaluación son la base para el planteamiento y/o replanteamiento de las estrategias didácticas y la detección de necesidades específicas de los estudiantes.

Por otra parte, es necesario conceptualizar qué es una contingencia. Respecto a esto se tiene que

...suele referirse a algo que es probable que ocurra, aunque no se tiene una certeza al respecto. La contingencia, por lo tanto, es lo posible o aquello que puede, o no, concretarse [...] Otra manera de entender la noción de contingencia es como un acontecimiento cuya realización no está prevista. Una contingencia, en este sentido, puede ser espontánea o estar provocada [Pérez y Merino, 2015, s.p.].

La contingencia en México fue derivada de una emergencia sanitaria ocasionada por el virus SARS-CoV-2, anunciada por las autoridades gubernamentales del país, con fecha 30 de marzo del 2020, publicada en el Diario Oficial de la Federación. En condiciones de normalidad las clases y la evaluación son predecibles y estables; en la contingencia las personas, eventos y planes son impredecibles, con un nivel de incertidumbre significativamente más alto de lo normal.

En una situación de contingencia, todos los actores de la escuela y los padres de familia se ven afectados por la incertidumbre y actos que transforman abruptamente los procesos escolares normales. Con estas condiciones las estrategias de enseñanza y la evaluación tiene que adecuarse a las circunstancias de la situación de emergencia. 


\section{Paradigma PRESENCIAL Versus PARAdigma A Distancia}

En México y el mundo, desde los inicios del 2020 las escuelas cerraron sus puertas y la actividad escolar se realizó a distancia; por lo general, en los hogares del alumnado las formas de trabajo se combinaron en acciones sincrónicas y asincrónicas; las primeras son las sesiones de clase por medios electrónicos, las segundas son acciones que los alumnos puedan realizar las actividades en cualquier horario, que dependerán de las condiciones y posibilidades de cada estudiante.

La enseñanza a distancia y la enseñanza presencial poseen estructuras distintas, aunque comparten la esencia de la acción, el logro de los aprendizajes de los estudiantes. Es en esta parte en la que la evaluación cobra sentido muy especial en la detección de las necesidades de los alumnos para la construcción de los conocimientos, en el desarrollo de las capacidades, de contenencias o la solución de problemas específicos preparados para la formación de la persona.

El paradigma presencial se caracteriza por la presencia de los estudiantes en un tiempo y espacio determinados. Implica una interacción cara a cara entre estudiantes con sus compañeros y con el docente. Implica el uso de materiales e infraestructura escolar, como libros, tecnología, conectividad, espacios de apoyo académico, salones de clase, espacios deportivos, etcétera; pero sobre todo la presencia física del docente con el alumnado.

Esa presencia del profesor/a es fundamental para el planteamiento de la dinámica en la que se desarrolla el aprendizaje. Los procesos se hacen más directos y en lo general más fáciles, tanto para el maestro como para los estudiantes. Se tiene la posibilidad de atender los problemas de manera más inmediata.

En el paradigma de educación a distancia, mediante la actividades sincrónicas y asincrónicas se combina una serie de estrategias didácticas en consideración de las condiciones sociales, económicas, psicológicas y académicas; en este sentido las acciones se deben hacer más diversificadas, necesariamente bajo una perspectiva humanista, como lo plantean Arias y Molano (2016).

El enfoque humanista, según Arias y Molano (2016), se caracteriza como

...la formación humanista en la escuela mantiene vigencia porque lo que se juega es la condición humana y las posibilidades de construir sociedades democráticas. En tal sentido, convienen análisis reposados de las prácticas, los sujetos y los discursos que imperan en la actualidad a la luz de un referente humanista que procura cualificar la sociedad y dignificar al ser humano [p. 7].

En este sentido se requiere, sobre todo en los tiempos de contingencia y de incertidumbre para maestros y estudiantes, que este enfoque humanista invada las acciones didácticas, porque con ello se atenderá al alumnado con mayor sensibilidad hacia las condiciones en que desarrollan sus actividades escolares, tomando en consideración las posibilidades no solo personales de los estudiantes, sino también las limitaciones o barreras socioeconómicas y familiares. 
En este paradigma de trabajo a distancia se requiere, como señalan Dávalos y Soto (2020), que, para el desarrollo de las actividades escolares, los docentes, directivos y autoridades educativas reflexionen sobre los cuestionamientos de la figura 1.

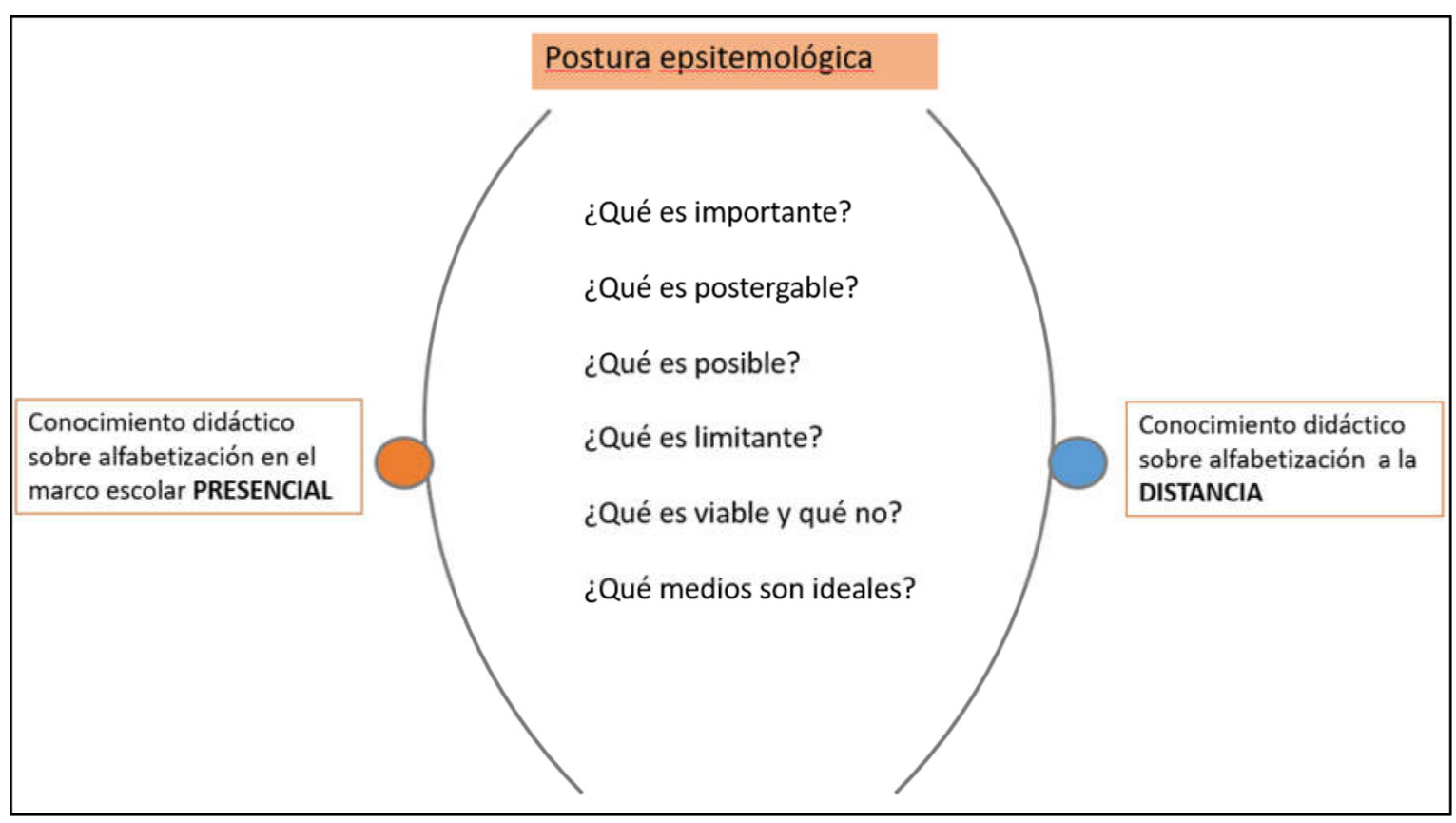

Figura 1. Reflexión sobre el trabajo escolar presencial y a distancia.

Fuente: Dávalos, 2020.

Las preguntas que se plantean en la figura 1 son detonadoras de toma de decisiones de los docentes al momento de planear sus actividades de enseñanza, pero también lo son de los directivos de las escuelas y de las autoridades educativas para que creen las condiciones necesarias para el desarrollo de las acciones en tiempos de contingencia y de incertidumbre.

En consideración de la reflexión dirigida por estas preguntas, la evaluación de los aprendizajes se transforma para ser coherente con los cambios en la forma de realizar los procesos de enseñanza y de aprendizaje, priorizando lo que debe evaluarse, el enfoque de hacerlo y la consideración de las condiciones personales y contextuales de los y las estudiantes.

\section{Ámbitos SOCIAL y ECONÓMico}

La situación de contingencia por la emergencia sanitaria ha impactado en las actividades escolares y en las posibilidades de participación en ellas. Esto se traduce en que en el alumnado existe diversidad de condiciones sociales y económicas para el desarrollo de los procesos escolares en la modalidad a distancia. 
Existen familias cuyos sostenes económicos perdieron sus empleos o se redujo considerablemente su ingreso. Esta condición se extiende directamente hacia las posibilidades de tener implementos tecnológicos y de conectividad para atender de una mejor manera la educación a distancia.

El número de personas que hacen uso de las tecnologías en una familia y la disponibilidad de ellas es uno de los elementos en los que se ha observado problema. Existen familias que tienen tres o cuatro hijos que requieren computadora o teléfono para ingresar a las actividades escolares, y a veces no cuentan con esa tecnología o solo tienen uno o dos equipos, lo que obliga a que se turnen el uso de los dispositivos. A esto se le agrega el hecho de que no tienen conectividad o es muy limitada.

Estas situaciones propician condiciones socioemocionales especiales que son proclives a provocar la no-participación en las actividades, a participar con menor motivación, a veces con frustración; también es posible que generen problemas de interacción entre los mismos miembros y que produzcan tensiones entre los miembros de la familia, lo que agrava la situación general (Robles Morales, Antino, De Marco y Lobera, citados en Trujillo, Fernández, Segura y Jiménez, 2020).

Estas situaciones, como lo señalan Trujillo et al. (2020),

presentan un conjunto heterogéneo de situaciones, aunque distintos indicadores señalan carencias en alfabetización mediático-informacional [...] y un problema de desigualdad no solo derivada por el acceso a la tecnología (según el INE, el 91,2\% de los hogares tienen conexión de banda ancha) [p. 5].

Esto da una idea clara de cómo los aspectos sociales y económicos impactan directamente en las posibilidades de atender el proceso educativo a distancia y a tener las condiciones óptimas para ello en el ámbito de las emociones que disponen o predisponen al estudiantado para cumplir con sus compromisos escolares.

Con lo anterior se observa que la situación para el desarrollo de las actividades de aprendizaje es muy diversa para los y las estudiantes. A cada uno de ellos le exige algo diferente, en los casos más desfavorecidos las condiciones adversas se les acumulan. En el contexto de la evaluación del aprendizaje Trujillo et al. (2020) plantea la problemática así:

...este escenario coloca al alumnado en situación socioeconómica más desfavorecida en clara desventaja respecto a la evaluación: pueden existir dificultades para el acceso a internet en algunos hogares, como también puede haber dificultades por parte de las familias para ayudar a sus hijos e hijas en esta situación. Por otro lado, es importante considerar las posibles situaciones adversas que se pueden estar viviendo en los hogares (estrés, ansiedad, problemas de salud, problemas laborales y económicos, ausencia de adultos por motivos laborales, etc.) y el impacto negativo que esto puede tener en el aprendizaje y la realización de tareas por parte del alumnado, especialmente aquel que se encuentre en situación desfavorecida [p. 23]. 
Para cerrar este pequeño apartado sobre el impacto socioeconómico en la educación y especialmente en la evaluación de los aprendizajes, es importante reflexionar que el estudiantado, vulnerable y vulnerado por las condiciones de la contingencia sanitaria, claramente se encuentra en una desventaja para el desarrollo de las actividades escolares y, por consiguiente, de tener un proceso de evaluación justo, que sea congruente con las condiciones personales de cada uno de ellos.

\section{EVALUACIÓN PARA EL APRENDIZAJE}

En la contingencia sanitaria, los procesos de enseñanza y aprendizaje se transforman para ajustarse a las circunstancias, necesidades y posibilidades de las instituciones y del alumnado. La evaluación, como parte de este proceso, no es la excepción, y no solo eso, sino que es considerada como el elemento fundamental para definir la continuación de los estudios para las personas más vulnerables y vulneradas por las condiciones económicas y socioemocionales derivadas de la situación crítica de incertidumbre e imposibilidad de realizar las actividades de aprendizaje que realizaban en los tiempos de la normalidad, anterior al mes de marzo del año 2020 (para México).

En estas circunstancias educativas críticas es donde cobra sentido el hecho de evaluar para mejorar el aprendizaje y para dar oportunidades de sostener un sistema educativo que se encuentra en medio de una situación inesperada, que no tiene la intención de perjudicar al estudiantado de los diversos niveles educativos. Sin embargo, se han observado acciones aisladas de instituciones y docentes que no han tenido la plena conciencia de la problemática originada por la emergencia sanitaria y los problemas socioeconómicos y socioemocionales, actuando con criterios de evaluación inflexibles, que dañan principalmente a los estudiantes más desfavorecidos.

Con base en esta idea, es necesario plantear una evaluación con un sentido más formativo, como lo señalan Sánchez y Martínez (2020), que establecen su utilidad para dar seguimiento al avance del estudiante en su aprendizaje para retroalimentar e intervenir sobre sus logros, proponiendo que esta actividad evaluativa sea permanente durante el proceso de enseñanza, no solo al final de este, porque no habrá tiempo para atender las deficiencias encontradas.

Por lo tanto, la evaluación para el aprendizaje debe cambiar de ser un proceso de acreditación y certificación, como señala Nápoles (2020), a un proceso de acompañamiento grupal e individual que permita tener información en el mejor momento para apoyar la intervención docente y asegurarse de que el alumnado está aprendiendo. La calificación derivada de la evaluación debe ser solo una actividad administrativa final de cada bloque de trabajo, con propósito certificatorio, pero la evaluación se propone como una acción permanente del maestro. Esto se ha convertido en una tarea compleja, según lo explica Ruiz (2020): 
La evaluación se ha convertido en una tarea compleja para el docente, pues en el contexto actual derivado del problema de salud pública, ahora la evaluación formativa y sumativa se ha tenido que adecuar a través de ambientes virtuales, donde el docente tiene que realizar el seguimiento del alumno en el que los procesos de enseñanza-aprendizaje son más difíciles de observar y valorar [p. 3].

Para atender esta situación problemática que plantea la evaluación es necesario que tanto las autoridades educativas, los directivos y los docentes de las escuelas participen activamente con plena conciencia de que los efectos socioeconómicos y socioemocionales actúan en detrimento de la posibilidad de aprender, por efecto de las limitaciones de recursos económicos y tecnológicos y de las condiciones personales que se ven afectadas por la situación de encierro. En estos casos la flexibilidad y las adecuaciones en los contenidos, metodologías y propósitos educativos de evaluación son elementos que coadyuvan a generar condiciones más incluyentes y de mayor certidumbre ante los efectos de la contingencia que se vive. La atención al bienestar personal de los estudiantes se vuelve un asunto prioritario de las instancias educativas en lo general y de los docentes en particular.

\section{Problemas Que Plantea la eValuación emergente}

La equidad en uno de los asuntos más importantes que deben atenderse cuando se trata de una contingencia, porque no debe considerarse que todo el estudiantado tiene las mismas oportunidades de acceso a las formas de educación a distancia. Los problemas que se presentan son muy variados y requieren de análisis especial en el contexto en que se dan.

Trujillo et al. (2020) plantean una serie de problemas que son dignos de considerar por los docentes:

- Existen enormes dificultades para la calificación justa y eficaz en un contexto virtual.

- Se requieren cambios en los criterios de evaluación y promoción legalmente establecidos, de tal forma que el profesorado tenga un marco legal al cual atenerse.

- Existe un problema de tiempos y plazos para una adaptación rigurosa del currículo, lo cual implica un trabajo de coordinación intra- e intercentros que puede ser complicado o imposible en la situación actual.

- Un número amplio de opiniones afirman que el rango de calificación presente en la normativa no se adapta a la situación actual, mostrándose especialmente en desacuerdo con la posibilidad de un suspenso que se derivara de la imposibilidad de conectarse a la docencia en línea u otras situaciones de desigualdad por motivos socioeconómicos, familiares o incluso sanitarios [p. 24]. 
A esta lista se le agregan las condiciones de ansiedad, falta de salud propia o de algún familiar, economía precaria, falta de tecnología, falta de conectividad, necesidades de trabajar para contribuir a la economía familiar, exceso de esfuerzo para atender las actividades de aprendizaje, entre otras.

Estas situaciones implican soluciones individuales y colectivas de los docentes y directivos de las escuelas de los diferentes niveles educativos. Estas soluciones deben diversificarse para atender la mayor parte de las necesidades generadas por condiciones individuales de los y las alumnas. Para ello, las acciones concretas de inclusión y justicia se deben poner por encima de los criterios normativos de evaluación.

\section{Ajustes a la evaluación en la contingencia}

Para continuar con los procesos educativos marcados por los tiempos y programas establecidos en los diferentes calendarios escolares de todos los niveles del sistema educativo nacional, se hicieron ajustes que permitieran continuar con las actividades escolares desde los hogares de estudiantes y maestros. Al respecto, Nápoles (2020) comenta que

El Covid-19 obligó a modificar hábitos, la virtualización, nos hizo repensar y replantear el proceso. Desde un principio, se plantearon tres objetivos clave: primero, garantizar la continuidad del ciclo lectivo; segundo, sortear las desigualdades socioeconómicas de los 11,5 millones de jóvenes que hoy intentan seguir adelante con el aprendizaje desde sus hogares; y tercero, descubrir e implementar nuevas formas y modelos educativos que no sean meramente circunstanciales, sino una oportunidad para seguir profundizando y desarrollándolos cuando pase la pandemia [p. 18].

En un principio, los ajustes a las actividades escolares se realizaron pensando que la contingencia tardaría uno o dos meses, tal vez tres. Sin embargo, conforme fue pasando el tiempo se tuvieron que extender las condiciones especiales, no sólo del desarrollo de los cursos, sino la evaluación, acreditación y certificación, toda vez que este problema se presentó en el semestre en el que generalmente se cierran ciclos escolares. Hubo generación que concluyó el preescolar, primaria, secundaria, bachillerato, educación superior e incluso posgrados.

Todos los egresados de los niveles educativos vivieron una serie de incertidumbres relacionadas con el estatus de certificación de sus estudios. Posteriormente se tuvo que ver la posibilidad de que estos estudiantes ingresaran a los niveles subsiguientes o se incorporaran a sus ejercicios laborales quienes concluyeron carreras formativas.

Durante ya casi un año de actividades educativas a distancia, se han identificado acciones específicas que han permitido continuar con el desarrollo de los programas educativos; para lograrlo, las instituciones tuvieron que hacer algunas reconsideraciones a los procesos cotidianos de enseñanza y aprendizaje. Con respecto a esto, Trujillo et al. (2020) explican estos cambios en cuatro escenarios o tipos distintos de ajustes institucionales (ver figura 2). 
ESCENXRIOS SEGƯN LX ESCXIX DE XSUNCION DE RESPONSXBIIIDXD
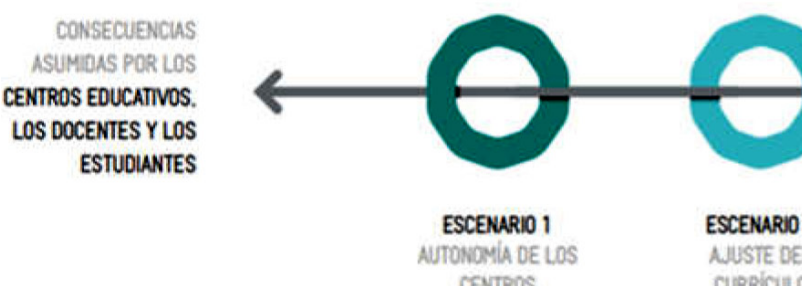
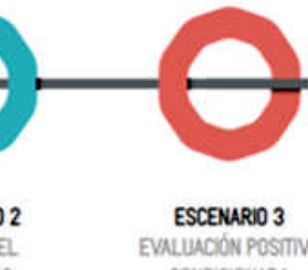

conoiciovan
CONSECUENCUS

ASUMIOAS POR

EL ESTADO

Figura 2. Cuatro escenarios o tipos de ajustes educativos realizados por las escuelas.

Fuente: Trujillo, Fernández y Jiménez, 2020, p. 6.

La descripción de los escenarios explica las acciones realizadas por las instituciones, considerando diferentes niveles de responsabilidad asumida entre los docentes y la escuela, en contraste con la que asume el Estado y el sistema educativo nacional:

El primero de los escenarios podríamos denominarlo "autonomía de los centros" y representa un planteamiento de toma de decisiones mínima por parte de la Administración, que optaría porque sean los centros quienes resuelvan el problema de la evaluación. El segundo escenario, denominado "ajuste del currículo", supone una toma de decisiones de nivel medio para solucionar los problemas de justicia y equidad respecto a la evaluación, pero dentro del ámbito de actuación de los centros educativos. El tercero es un escenario denominado "evaluación positiva condicionada" y representa un nivel de toma de decisiones alto por parte de la Administración para afrontar la evaluación del curso 2019-2020. Finalmente, el cuarto escenario, denominado “evaluación positiva sin condiciones", contempla la promoción de todo el alumnado y la búsqueda de soluciones educativas a lo largo del curso 2020-2021 [Trujillo, Fernández y Jiménez, 2020, p. 6].

Los cuatro escenarios se plantean una diversidad en la flexibilidad del centro educativo y los docentes para atender el problema de la evaluación. Gradualmente los escenarios planteados van proponiendo formas de atender la problemática, desde el ejercicio de la autonomía otorgada a las escuelas, el ajuste al currículo, la evaluación positiva con condiciones, hasta el escenario de la evaluación positiva sin condiciones, en la que se pretende aprobar a los alumnos o no retenerlos por falta de evidencias de sus avances en los aprendizajes.

En las instituciones del sistema educativo mexicano se implementaron indistintamente los cuatro escenarios referidos, no todos juntos ni al mismo tiempo, sino que fueron adoptándose como medidas específicas en respuesta a las necesidades que se planteaban desde la puesta en práctica de la educación a distancia en consideración con las posibilidades de los estudiantes para atender las actividades escolares en confinamiento y de la realización de la calificación, acreditación y certificación escolar, evitando reprobaciones por efecto de las inequidades existentes para atender las exigencias educativas en el esquema obligado de educación a distancia. 
Los dos últimos escenarios (evaluación positiva con y sin condiciones) fueron los de los niveles de preescolar y primaria, a partir la educación secundaria las exigencias se incrementaban hasta llegar a la educación superior, en la cual se presentaron ajustes evaluativos con menor nivel de flexibilidad, según refieren Trujillo et al. (2020), quienes exponen que "se observan correlaciones directas del género con los escenarios 1 y $2(r=0,09, p<0,01 ; r=0,04, p<0,05)$ e inversas con el escenario $4(r=-0,12, p$ $<0,01)$, lo cual confirma las diferencias en las preferencias de escenarios 1 y 2 para el género masculino y del escenario 4 para el género femenino” (p. 17). De acuerdo con esta cita, las maestras son más proclives a ser flexibles en la evaluación, con preferencia del escenario 4 (evaluación positiva sin restricciones), mientras que los docentes varones tuvieron la tendencia a preferir los escenarios 1 y 2 , relacionados con la autonomía del centro y los ajustes a los contenidos curriculares.

En términos de equidad y justicia, para los estudiantes más desfavorecidos los criterios del escenario 4 son los más incluyentes, toda vez que permite la promoción de los y las estudiantes con mayores problemas para realizar las actividades escolares propuestas, principalmente cuando se trata de sesiones sincrónicas virtuales.

\section{CONCLUSIÓN Y RECOMENDACIONES PARA EVALUAR}

Para atender la problemática de la evaluación se presentan recomendaciones que fueron compiladas de diferentes fuentes de consulta, así como aportaciones propias, bajo la sugerencia de integrar estos elementos en un esquema holístico de atención, en los ámbitos individual, grupal y escolar.

La primera serie de recomendaciones es aportada por Sánchez y Martínez (2020), tiene que ver con que los cuerpos colegiados académicos desarrollen un plan de evaluación que, en lo más posible, coincida con lo planteado en los planes y programas de estudio y en específico con cada área del conocimiento establecidos en los programas; la validación de cualquier evidencia de aprendizaje desarrollada por el estudiantado; orientar la evaluación "para el aprendizaje", no solo "del aprendizaje"; evaluar durante el proceso (evaluación formativa y procesual), no solo al final; considerar que cualquier técnica o instrumento es un medio, no un fin en sí mismo para la evaluación; realizar evaluación para potenciar el aprendizaje, cuestionamientos analíticos y reflexivos; desarrollar procesos evaluativos incluyentes evitando todo tipo de discriminación; establecer códigos éticos para el desarrollo de las evaluaciones y la presentación de las evidencias del estudiantado; usar la evaluación para mejorar el aprendizaje; promover exámenes a libro abierto y variedad de instrumentos, y promover la autoevaluación y la coevaluación.

La segunda serie de recomendaciones se encontró en Nápoles (2020), implica:

...establecer metas específicas y realistas para cada lección y explique cómo se evaluará el aprendizaje; intercalar dos o tres preguntas de evaluación periódicamente durante la instrucción en línea; 
utilizar una cantidad suficiente de preguntas para determinar si los alumnos han cumplido las metas de cada lección; tomar decisiones sobre la instrucción con prontitud después de recopilar la información de la evaluación; al regresar a la escuela, usar la información de las evaluaciones sumativas, si está disponible, para describir el progreso del estudiante durante COVID-19 y el aprendizaje en el hogar [...] la socioformación como el enfoque que oriente la evaluación que realiza el docente en un contexto educativo actual, siendo fundamental el uso de la taxonomía que permita determinar el nivel de desempeño logrado por los estudiantes a través de ambientes educativos virtuales y el cambio en la metodología de evaluación debe generar que los estudiantes resuelvan problemas del contexto que les tocó vivir y desde ahí promuevan una mejora en su calidad de vida [p. 21].

La tercera serie de recomendaciones son una aportación propia, fundamentada en la experiencia vivida en este proceso de contingencia como docente de una escuela Normal rural, en la que las estudiantes pertenecen a las clases sociales más desfavorecidas y muchas de ellas viven en contextos marginados. Esta aportación implica: la consideración de que el principio de la evaluación en los grupos escolares es mejorar el aprendizaje de las estudiantes; tener como objetivo la garantía de la atención al estudiantado para su desarrollo integral y la generación de condiciones humanas más adecuadas a sus necesidades sociales y personales; el establecimiento de procesos incluyentes en la enseñanza y en la evaluación de los aprendizajes; la realización de adecuaciones administrativas relacionadas con la enseñanza y el aprendizaje: que la norma no represente una barrera para el desarrollo de las actividades escolares en condiciones extraordinarias de contingencia; la identificación de barreras para el aprendizaje, eliminarlas y evitar que afecten los procesos de evaluación; el establecimiento de procesos de evaluación idiográfica, en el sentido planteado por Casanova (1998), para atender el desarrollo del aprendizaje en términos individuales, especialmente de los y las estudiantes más desfavorecidos por las condiciones contextuales y personales, los más vulnerados en los ámbitos socioeconómico y socioemocional; realizar cursos y talleres para docentes en el campo de la evaluación incluyente y contextualizada; establecer criterios de validez de evaluación no-discriminatorios ni ventajosos para el estudiantado más favorecido económica, social y personalmente; que la posesión de herramientas tecnológicas y de conectividad no sea lo que establezca la diferencia entre los aprendizajes y la evaluación del estudiantado; la flexibilidad en la aceptación de las evidencias de aprendizaje, toda vez que el estudiantado tiene diversidad de posibilidades o restricciones tecnológicas, las cuales no debe ser la diferencia en la calificación de sus trabajos; flexibilidad en los tiempos de aceptación de tareas, sobre todo, por el hecho de otorgar calificación por entrega a tiempo de trabajos, que implica considerar al tiempo como criterio de evaluación, lo que la hace inválida, y discrimina por las posibilidades tecnológicas y de conectividad del estudiantado más desfavorecido, y la consideración general de las condiciones contextuales del estudiantado para determinar los procesos de evaluación, en el sentido de que en tiempos de contingencia existe una serie de elementos que afectan las actividades escolares. 
En lo general, y como resumen, se recomienda una evaluación equitativa bajo un enfoque humanístico, que consiste en una evaluación sin discriminación, incluyente, sin ventajas para algunos estudiantes, idiográfica, válida para las condiciones de enseñanza y aprendizaje en contingencia, sincrónica y asincrónica, justa y flexible. Estas consideraciones se aplican también para los directivos y autoridades educativas, en lo que respecta a la toma de decisiones y solicitud de evidencias y otros elementos administrativos y de fiscalización del trabajo escolar.

Se tiene conciencia de que la evaluación estrictamente personalizada a profundidad es imposible si se tienen grupos numerosos, o si el tiempo disponible para ello es reducido, por ello se recomienda, en estos casos, realizar procesos evaluativos de aplicación grupal; con esta acción se identifican los casos que requieren un seguimiento individual, personalizado, para identificar o programar los ajustes específicos que ayudarán a que aprendan y se les evalúe solo lo más indispensable para atender los propósitos de cada unidad de logro o curso.

Con esta propuesta se privilegia la comunicación directa con el estudiantado, en lo más posible, para establecer una relación más estrecha de acompañamiento en el proceso de aprendizaje y de evaluación de los logros, y de los procesos para hacerlo.

En tiempo de contingencia la revisión de los referentes de evaluación es muy importante, ya que no todos los estudiantes pueden cubrir las intenciones educativas establecidas por el programa escolar. Tampoco se debe frenar el avance de los y las estudiantes que pueden avanzar más en los logros establecidos oficialmente, incluso los que podrían superarlos. La idea es que los alumnos/as que pueden desarrollar todos los contenidos del programa, así como los que pueden sobrepasarlos, no sean un punto de referencia para los que están en condiciones más desfavorecidas, sea cual fuere el motivo. También se requiere atender al grueso del alumnado sin desatender a los más vulnerables.

\section{REFERENCIAS}

Arias, D. H., y Molano, F. (2016). Escuela y formación bumanista: miradas desde la investigación educativa. Bogotá D.C.: Kimpres/Universidad de la Salle.

Casanova, M. (1998). La evaluación educativa. Escuela básica. México: Secretaría de Educación Pública.

Casanova, M. A. (2020). Evaluación formativa e inclusiva en línea: ¿Cómo evaluar el aprendizaje ante los retos de la pandemia por el Covid 19? En J. Luna-Nemecio (coord.), Memorias del Quinto Congreso Internacional de Evaluación Socioformativa (VALOR A-2020). Cuernavaca, México: Centro Universitario CIFE. Recuperado de: https://cife.edu. $\mathrm{mx} /$ recursos.
Dávalos, A., y Soto, A. (2020). Alfabetización en pandemia. Recuperado de: https:// sites.google.com/view/de$\mathrm{duc} /$ recursos-acad $\% \mathrm{C} 3 \% \mathrm{~A} 9$ micos?authuser $=0 \#$ h. rhuuznands $3 \mathrm{~m}$.

De la Oliva, D. (2020). La autoevaluación en las escuelas de educación primaria para la mejora de la calidad educativa y de la inclusión social. En J. Luna-Nemecio (coord.), Memorias del Quinto Congreso Internacional de Evaluación Socioformativa (VAlora-2020). Cuernavaca, México: Centro Universitario CIFE. Recuperado de: https://cife. edu.mx/recursos. 
Miller, A. (2020). Evaluación formativa en educación a distancia. Recuperado de: https://www.edutopia.org/article/ formative-assessment-distance-learning.

Nápoles, J. E. (2020). La evaluación en tiempos de pandemia. Educación Matemática 2020. II Congreso Virtual Iberoamericano de Formación de Profesores de Matemáticas, Ciencias y Tecnologías (II ConviBE-ForPro) UFRN 2020. Recuperado de: https://www.researchgate.net/publication/344442546_La_evaluacion_en_ tiempo_de_pandemia_Educacion_Matematica_2020.

Pérez, J., y Merino, M. (2015). Definición de contingencia. Recuperado de: https://definicion.de/contingencia/. Requena, M. (2020). La evaluación socioformativa de los aprendizajes en la educación basada en competencias. En J. Luna-Nemecio (coord.), Memorias del Quinto Congreso Internacional de Evaluación Socioformativa (VALORA-2020). Centro Universitario CIFE, Cuernavaca, México. Recuperado de: https://cife.edu. $\mathrm{mx} /$ recursos.

Ruiz, S. (2020). La evaluación en tiempos de pandemia. En J. Luna-Nemecio (coord.), Memorias del Quinto Congreso Internacional de Evaluación Socioformativa (V ALORA-2020). Cuernavaca, México: Centro Universitario CIFE. Recuperado de: https:/ / cife.edu.mx/recursos. Sánchez, M., y Martínez, A. (2020). Evaluación del y para el aprendizaje a distancia: recomendaciones para docentes de edu- cación media superiory superior. México: Universidad Autónoma de México. Recuperado de: https:/ / cuaieed. unam.mx/descargas/investigacion/Recomendaciones_Evaluacion_Educativa_a_distancia.pdf.

Tobón, S. (2020). La evaluación socioformativa en la educación en línea. En J. Luna-Nemecio (coord.), Memorias del Quinto Congreso Internacional de Evaluación Socioformativa (VALORA-2020). Cuernavaca, México: Centro Universitario CIFE. Recuperado de: https:/ / cife.edu.mx/recursos.

Trujillo, F., Fernández, M., Segura, A., y Jiménez, M. (2020). Escenarios de evaluación en el contexto de la pandemia por Covid-19: la opinión del profesorado. Recuperado de: https://www.santillanalab.com/recursos/INFORME_ESCENARIOS_EVALUACION_COVID19_1_1.pdf..

UNESCO-OIE [Organización de las Naciones Unidas para la Educación, la Ciencia y la Cultura-Oficina Internacional de Educación] (2013). Construcción de capacidades para la implementación curricular. En Herramientas de capacitación para el desarrollo curricular. Una caja de herramientas. Ginebra, Suiza: UNESCOOIE. Recuperado de: http://www.ibe.unesco.org/ fileadmin/user_upload/COPs/Pages_documents / Resource_Packs/TTCD/sitemap/Modulo_6/Modulo_6.html.

Cómo citar este artículo:

Viramontes Anaya, E., y Viramontes Campos, O. (2021). Evaluación en educación a distancia por confinamiento. IE Revista de Investigación Educativa de la REDIECH, 12, e1216. doi: 10.33010/ie_rie_rediech.v12i0.1216. 